\title{
A Fast Algorithm for High-Accuracy Frequency Measurement: Application to Ultrasonic Doppler Sonar
}

\author{
Hironori Susaki, Member, IEEE
}

\begin{abstract}
In an attempt to investigate the technical feasibility of a CW Doppler sonar, we have examined a method of measuring low velocities with a high-velocity resolution, or frequency resolution, by use of a simple circuit configuration employing digital signal processing technique. The following discussion presents the results of the investigation. In the measuring method described below, the fast Fourier transform (FFT) of undersampled data is calculated and the Doppler shift is obtained by searching for a peak frequency of the power spectrum. To achieve the intended frequency resolution of $1 \mathrm{~Hz}$ by FFT operation, measurement of data for a minimum measuring period of $1 \mathrm{~s}$ is essential. If the sampling frequency is set to $50 \mathrm{kHz}$, the number of samples obtained during the minimum measuring period of $1 \mathrm{~s}$ would amount to 50000 . This is not practical in light of the time required for the FFT operation. To overcome this problem, our new measuring method employs a decimation technique for reducing the number of samples down to 1024 while maintaining a frequency resolution of about $1 \mathrm{~Hz}$. This paper describes how the processing time can be drastically reduced to about 1/300th compared to the conventional technique by a combination of complex exponential functions, filtering and decimation, and thereby indicates the possibility of real-time CW Doppler data processing.
\end{abstract}

Index Terms-A/D, aliasing, CW, decimation, demodulation, Doppler, FFT, FIR filter, I/Q, sampling, sonar, spectrum zooming, undersampling.

\section{INTRODUCTION}

$\mathbf{T}$ HE DOPPLER sonar is a device for measuring the velocity of a vessel based on the Doppler effect of ultrasonic waves. It is particularly useful in monitoring and controlling the behavior of a huge vessel moving at low speeds for preventing a collision and hard contact with berthing facilities. In a typical demodulator of a conventional Doppler sonar, an analog mixer converts the received signal to an intermediate frequency (IF) and the resultant IF signal is subjected to fast Fourier transform (FFT) operation. To achieve an improved frequency resolution, we employed an undersampling technique using a sampling $\mathrm{A} / \mathrm{D}$ converter, instead of using the analog mixer to shift, or down-convert, the received signal, together with frequency spectrum zooming technique for processing a Doppler shift signal [1]. In this method, the frequency band of the desired signal was shifted so that the spectrum would be centered on the zero frequency point by using a digital signal processor (DSP) in which the desired signal was multiplied by

Manuscript received November 27, 2000; revised June 13, 2001.

The author is with the Research Laboratory, Furuno Electric Company, Ltd., Nishinomiya 662-8580, Japan (e-mail: susaki@furuno.co.jp).

Publisher Item Identifier S 0364-9059(02)01620-5. a complex exponential function, and the frequency resolution of $1 \mathrm{~Hz}$ was realized with a small number of data obtained by decimation, thereby achieving a velocity resolution of $1 \mathrm{~cm} / \mathrm{s}$ or better. In this paper, we describe how frequency shift, filtering and decimation techniques are combined to achieve simplification of hardware, a reduction in memory capacity using the DSP and an increase in processing speed.

\section{UNDERSAMPLING}

The use of a broad-band, high-speed sampling A/D converter incorporating a sample-and-hold amplifier is widespread in recent years [2], [3]. The sampling theorem states that the original input signal can be reconstructed from sampled signal data if the input signal is sampled at a frequency at least twice as high as the highest frequency in the input signal. However, the undersampling technique is now widely implemented for a bandpass signal, in which the input signal is sampled at a frequency lower than the minimum sampling frequency required by the sampling theorem [4]. The undersampling technique is also called as direct IF sampling, sampling mixer, digital frequency converter, super Nyquist sampling, digital baseband converter, IF down conversion, harmonic sampling, and bandpass sampling. The term "bandpass sampling" is commonly used in textbooks related to signal processing [5], [6].

Generally, if the original signal is undersampled, aliased frequency components would overlap, making it impossible to exactly reconstruct the original information. Overlapping of the aliased frequency components can be avoided, however, by properly selecting the sampling frequency, the bandwidth of the input signal, and the highest frequency in the input signal if the signal to be handled is a narrow-band signal. The frequency band of the desired signal which is modulated to a high carrier frequency can be shifted to a lower frequency band by undersampling, without using the mixer. While the input signal is sampled by using a single A/D converter in the method described in this paper, the signal is converted to a complex (I/Q: in-phase/quadrature) representation for improving the frequency resolution. Re [7] showed how to recover the bandpass signal through the minimum number of its samples. Rice and $\mathrm{Wu}$ [8] showed that the I-component can be computed by way of a Hilbert transform. The relationships between the sampling frequency and lower/upper bandedge frequencies of the spectral range were also presented. Rosenkranz [9] showed a generalized quadrature sampling method. 
(1)

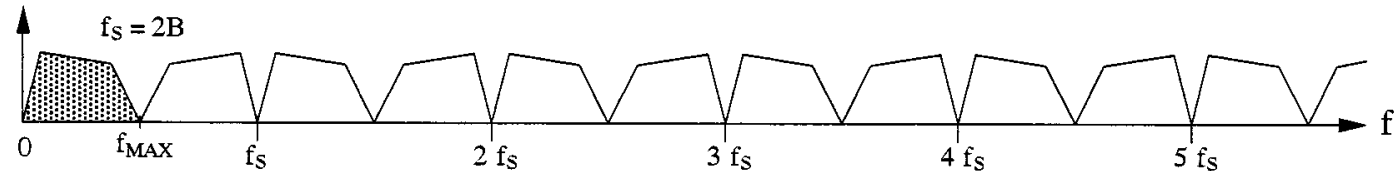

(2)

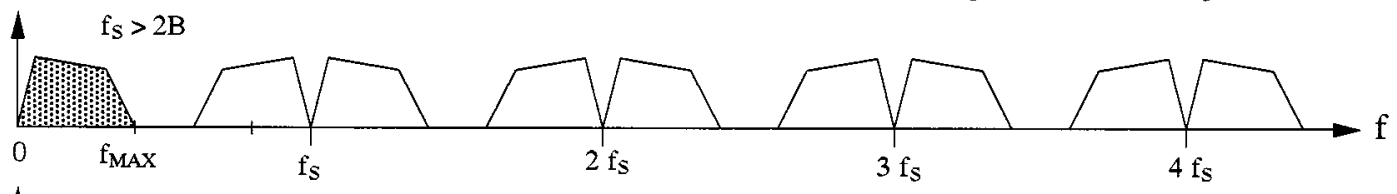

(3)

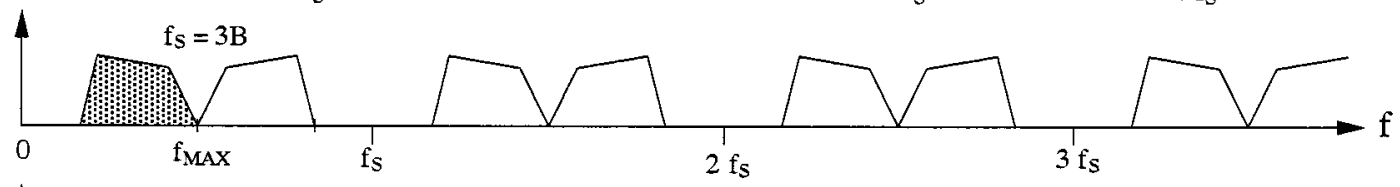

(4)

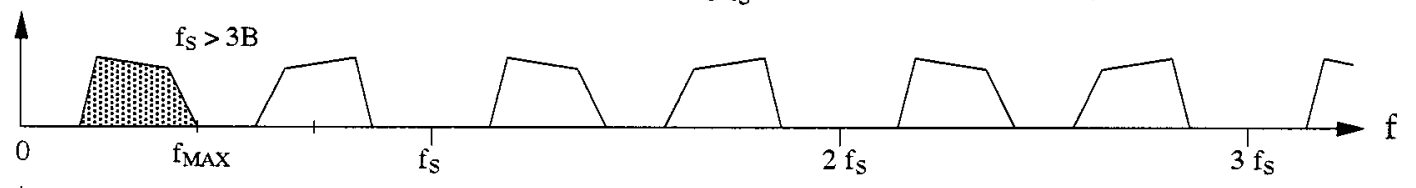

(5)

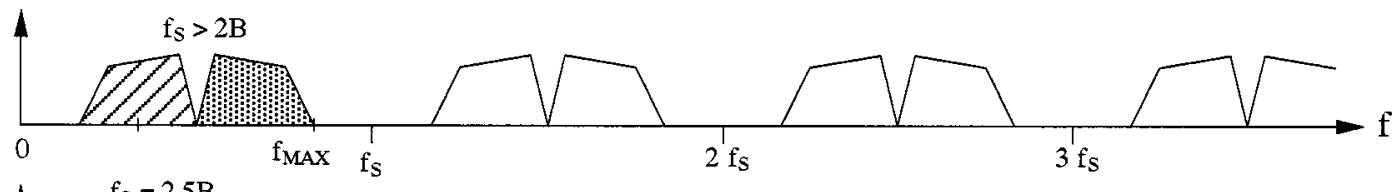

(6)

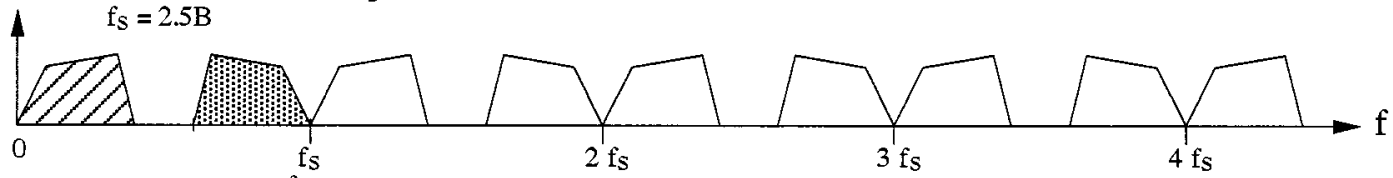

(7)

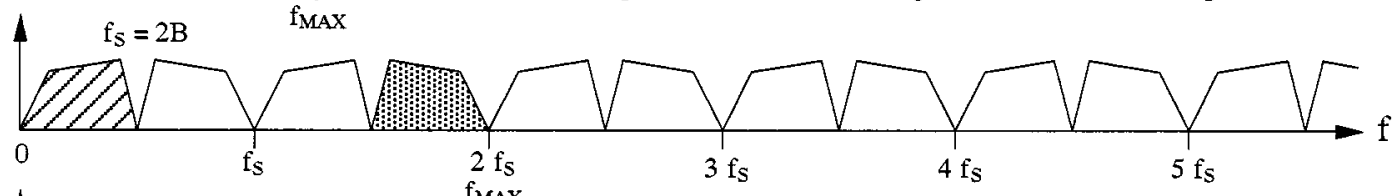

(8)

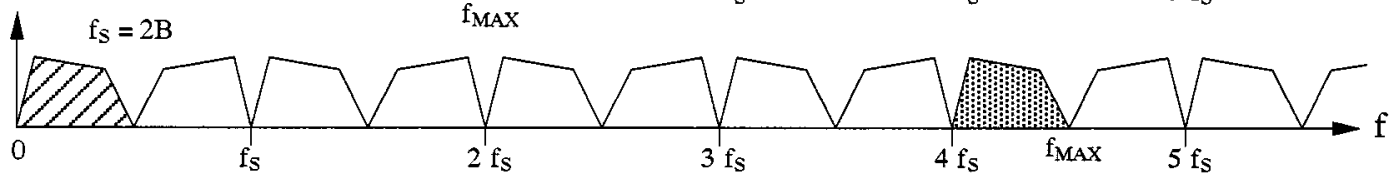

Fig. 1. Aliasing effect caused by real data sampling.

\section{A. Advantages of Undersampling}

A conventional receiver demodulator obtains a complex signal by using two analog mixers. This configuration has such problems as variations in the characteristics of individual analog components, fluctuation of the characteristics due to temperature changes, and their deterioration with the lapse of time. If the input signal is directly undersampled by a single A/D converter instead of the two analog mixers, it is possible to reduce the number of analog components, simplify the analog front-end circuit, and eliminate signal loss caused by the analog mixers. Furthermore, induction noise caused by dc offsetting can be eliminated, and leakage of the local frequency component to associated circuits can be avoided. Particularly significant advantages are that amplitude mismatching between I- and Q-signals is completely eliminated and phase deviation between the I- and Q-signals due to variations in the $\pi / 2$ phase shift can be disregarded.

\section{B. Relationship Between Input Signal and Sampling Frequency}

To clarify the relationship between the input signal and sampling frequency, Fig. 1 illustrates how aliased components are mapped by real data sampling under different conditions, in which $B$ is the bandwidth of the input signal, $f_{\mathrm{MAX}}$ is the maximum frequency in the input signal, and $f_{S}$ is the sampling frequency. The frequency band of the input signal is gradually changed to higher frequencies in Fig. 1(1)-(8) as depicted by shaded patterns.

Fig. 1(1)-(4) shows cases where the input signal is sampled at frequencies equal to or higher than twice the maximum frequency in the input signal. In these cases, the usable frequency band after sampling is same as the frequency band of the input signal. Fig. 1(5)-(8) illustrates cases where the input signal is sampled at frequencies lower than twice the maximum frequency in the input signal, in which the usable frequency band is shown by hatched patterns.

It would be noted from Fig. 1(5)-(8) that the aliased components well represent the input signal without any overlaps even when it is sampled at frequencies lower than twice the maximum frequency in the input signal.

In the cases of Fig. 1(5)-(7), the aliased components in the dc to $f_{S} / 2$ band are inverted in frequency with respect to the spectrum of the original signal. 


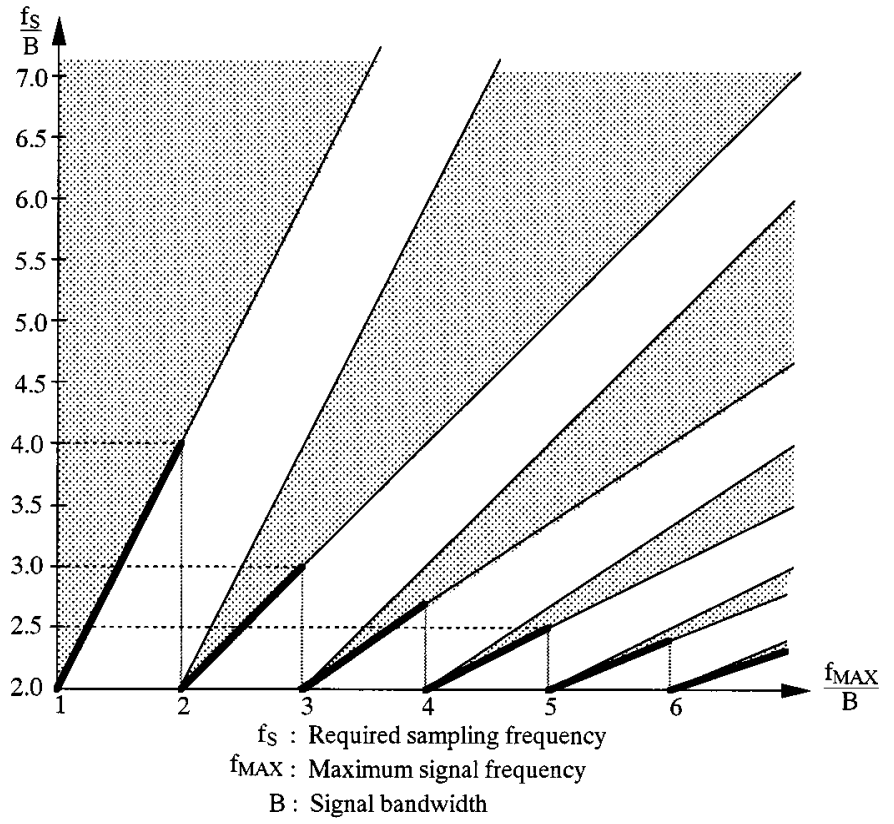

Fig. 2. Relationship between sampling frequency, signal bandwidth, and maximum signal frequency essential to sampling for a bandpass signal.

Fig. 1(6) shows the case in which the same input signal as depicted in Fig. 1(5) is sampled at $f_{S}=2.5 B$, where $f_{S}=$ $f_{\text {MAX }}$. Fig. 1(7) shows the case in which the center frequency of the input signal is increased and the sampling frequency is set to $f_{S}=2 B\left(f_{S}=f_{\mathrm{MAX}} / 2\right)$. Fig. 1(8) shows the case in which the center frequency of the input signal is further increased. In this case, the spectrum mapped in the dc to $f_{S} / 2$ band is an accurate reproduction of the original input signal, exhibiting no frequency inversion, even though $f_{S}=2 B$.

Whether the aliased components appearing in the dc to $f_{S} / 2$ band are inverted in frequency with respect to the frequency spectrum of the original signal is determined by the number of aliases that occur between the dc and the original signal. Frequency inversion occurs in the baseband alias if the number $N$ of aliases including the original signal itself is an even number, while no frequency inversion occurs if it is an odd number. When

$$
f_{\mathrm{MAX}}=N \cdot B, \quad N=1,2,3, \ldots
$$

the minimum sampling frequency can be set to $2 B\left(f_{S}=2 B\right)$.

Whether the frequency inversion occurs or not is determined in the system design stage in which the bandwidth of the input signal, the maximum frequency in the input signal, and the sampling frequency are defined. The frequency inversion does not pose a substantial problem, however. This is because whether it occurs or not is known in the system design stage and, even when it occurs, positive and negative frequency components of spectrum data can be easily inverted by software-based digital signal processing.

The relationship between the input signal bandwidth $B$, the maximum frequency $f_{\mathrm{MAX}}$ in the input signal, and the sampling frequency $f_{S}$ is depicted in Fig. 2 taking into account the foregoing discussion. The shaded areas including thick solid lines in Fig. 2 represent regions where allowable sampling operation for the band-pass signal can be performed.

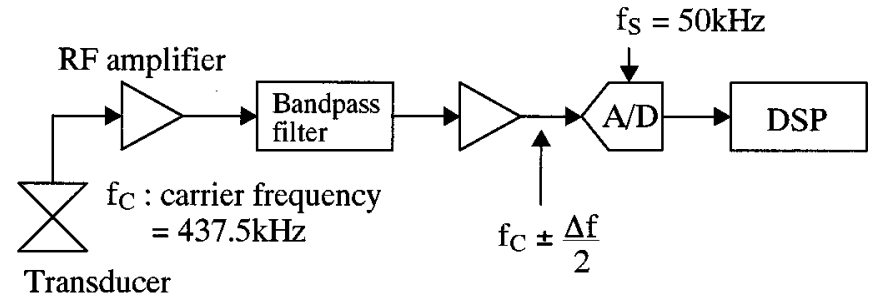

Fig. 3. Demodulator circuit of Doppler sonar.

The thick solid lines in Fig. 2 represent the minimum sampling frequency required for a bandpass signal [10]. To make the sampling frequency as low as possible, it is hereinafter assumed that

$$
f_{S}=2 B
$$

in this paper [11], [12]. Expressing the transmitting (carrier) frequency as $f_{C}$, we obtain

$$
f_{\mathrm{MAX}}=f_{C}+\frac{B}{2}
$$

When $N$ is an odd number, that is, when no frequency inversion occurs in the baseband alias [see Fig. 1(8)]

$$
f_{\mathrm{MAX}}=k f_{S}+B, \quad k=0,1,2, \ldots
$$

Thus, we obtain the following relationship:

$$
f_{S}=\frac{4 f_{C}}{4 k+1}, \quad k=0,1,2, \ldots
$$

When $N$ is an even number, that is, when the frequency inversion occurs,

$$
f_{\mathrm{MAX}}=k f_{S}, \quad k=1,2,3, \ldots
$$

However, if we use the following equation such that the value of $k$ begins from zero [see Fig. 1(7)]:

$$
f_{\mathrm{MAX}}=(k+1) f_{S}, \quad k=0,1,2, \ldots
$$

Then, we obtain the following relationship:

$$
f_{S}=\frac{4 f_{C}}{4 k+3}, \quad k=0,1,2, \ldots
$$

\section{PARAmeter Settings FOR CW DopPler SONAR}

Taking into account the aforementioned relationships, we consider a sonar system operating on a transmitting frequency of about $450 \mathrm{kHz}$. To satisfy the relationships given by (1), (2), and (8) above, it is here assumed that maximum input signal frequency $f_{\mathrm{MAX}}=450.0 \mathrm{kHz}$, transmitting frequency $f_{C}=437.5 \mathrm{kHz}$, input signal bandwidth $B=25.0 \mathrm{kHz}$, and sampling frequency $f_{S}=50.0 \mathrm{kHz}(=2 B)$.

Shown in Fig. 3 is an example of a receiver demodulator circuit of a CW Doppler sonar.

Fig. 4 shows the frequency spectrum of the received echo signal. 


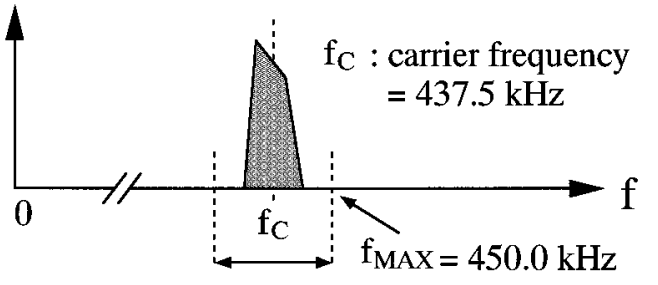

B: Bandwidth $=25.0 \mathrm{kHz}$

Fig. 4. Spectrum of received echo signal.

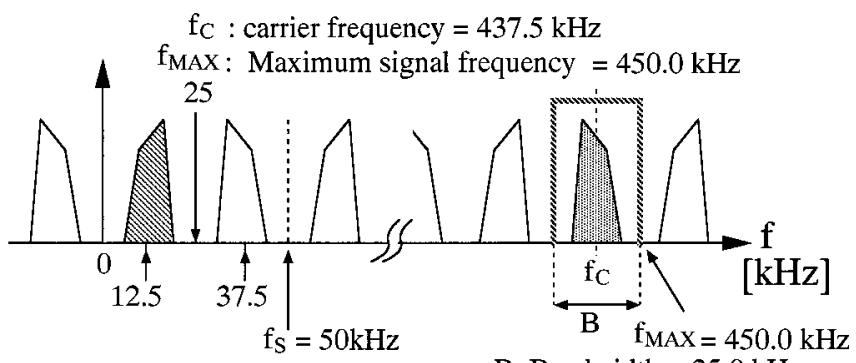

Fig. 5. Frequency spectrum after undersampling.

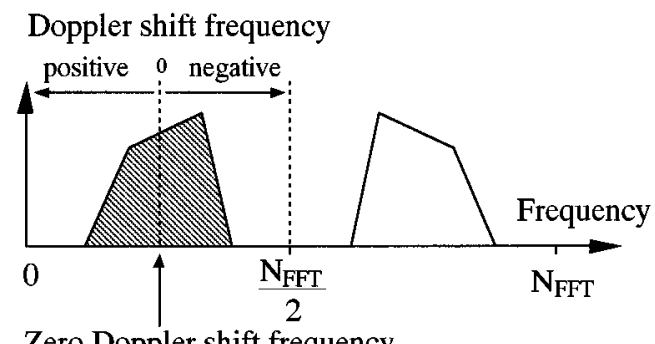

Zero Doppler shift frequency

Fig. 6. Discrete frequency spectrum obtained by FFT.

When the signal shown in Fig. 4 is digitized by the undersampling operation, a frequency-inverted spectrum appears in a frequency range of 0.0 to $25.0 \mathrm{kHz}$ as depicted in Fig. 5 since the number of aliases $N=18$, which is an even number.

The digital signal produced by the above undersampling process is entered to the DSP and the FFT operation is performed on $N_{\text {FFT }}$ samples. The result is a discrete frequency spectrum as shown in Fig. 6.

It is to be noted that discrete frequency $N_{\mathrm{FFT}} / 4$ corresponds to the zero Doppler shift frequency. Since the frequency spectrum is inverted, discrete frequencies 0 to $N_{\mathrm{FFT}} / 4$ correspond to positive frequencies while discrete frequencies $N_{\mathrm{FFT}} / 4$ to $N_{\text {FFT }} / 2$ correspond to negative frequencies.

To achieve the intended frequency resolution of $1 \mathrm{~Hz}$, the measuring period $T$ for obtaining the input data to be subjected to the FFT operation must be at least $1 \mathrm{~s}$. This means that it is essential to constantly store the sampled data for a period of $1 \mathrm{~s}$ or more. In other words, if it is desired to achieve a frequency resolution of $1 \mathrm{~Hz}$ or better from $2^{n}$ samples (where $n$ is an integer), the FFT operation must be performed on $65536\left(=2^{16}\right)$ samples per second since the sampling frequency $f_{S}$ is $50.0 \mathrm{kHz}$. More precisely, the frequency resolution achieved when the number of FFT samples is 65536 is $f_{S} / 65,536=0.76 \mathrm{~Hz}$.

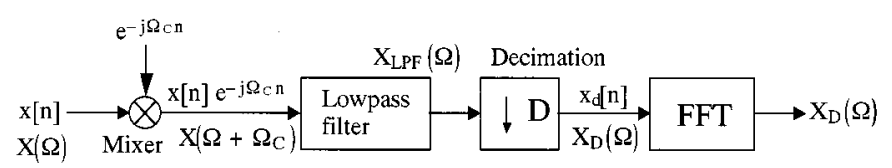

Fig. 7. Frequency spectrum zooming.
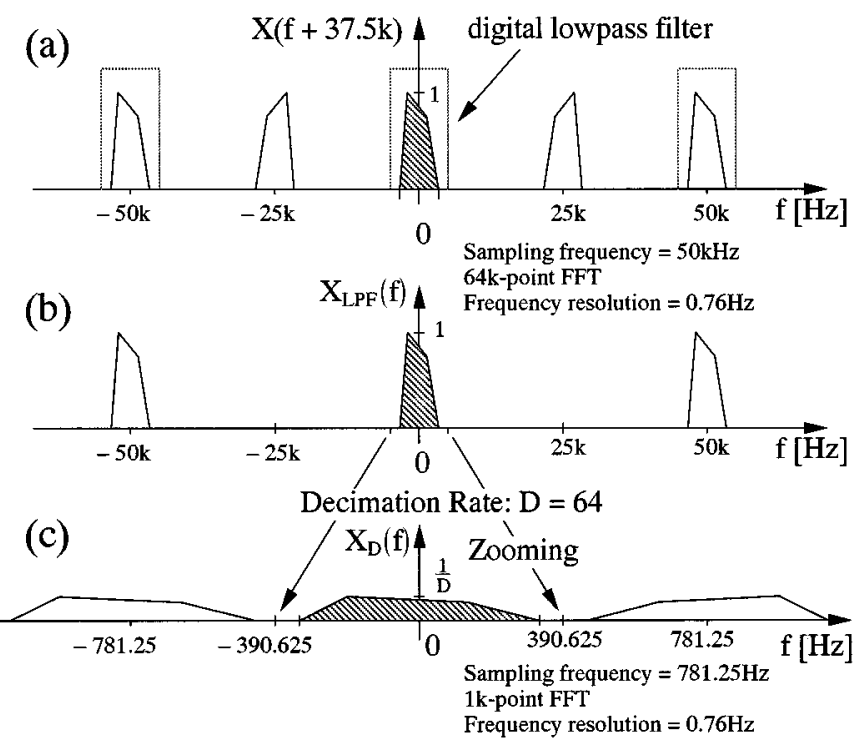

Fig. 8. Spectrum zooming by decimation. (a) The spectrum obtained after the spectrum inversion has been eliminated by $37.5-\mathrm{kHz}$ frequency shift. (b) The spectra obtained after lowpass filtering. (c) The spectra obtained after subsequent decimation.

\section{FREQUENCY SPECTRUM ZOOMING}

It is impractical in actual applications to perform the FFT operation on 65536 samples or more in the light of computation time and memory capacity. Here, the frequency spectrum zooming technique is employed to reduce the number of samples to be subjected to the FFT operation while maintaining the same frequency resolution as would be achieved by performing the FFT operation on the 65536 samples.

When the number of FFT samples is reduced by decimation, the spectrum is expanded about its zero frequency point. Thus, if the $12.5 \mathrm{kHz}$ point of the spectrum shown in Fig. 5, which corresponds to the zero Doppler shift frequency point, is shifted to the zero frequency point of the spectrum and sample data thus shifted is decimated, the desired frequency spectrum is expanded. Here, we consider a case where a spectral range centered on $37.5 \mathrm{kHz}$ is shifted by $37.5 \mathrm{kHz}$ to eliminate spectrum inversion.

\section{A. Frequency Spectrum Shifting}

The frequency spectrum obtained by the Fourier transform of a sequence of data $x[n]$ sampled at the sampling frequency $f_{S}$ is given by

$$
X(\Omega)=\sum_{n=-\infty}^{\infty} x[n] e^{-j \Omega n}
$$

Given the discrete complex exponential function $c[n]$

$$
c[n]=e^{-j \Omega_{C} n}
$$




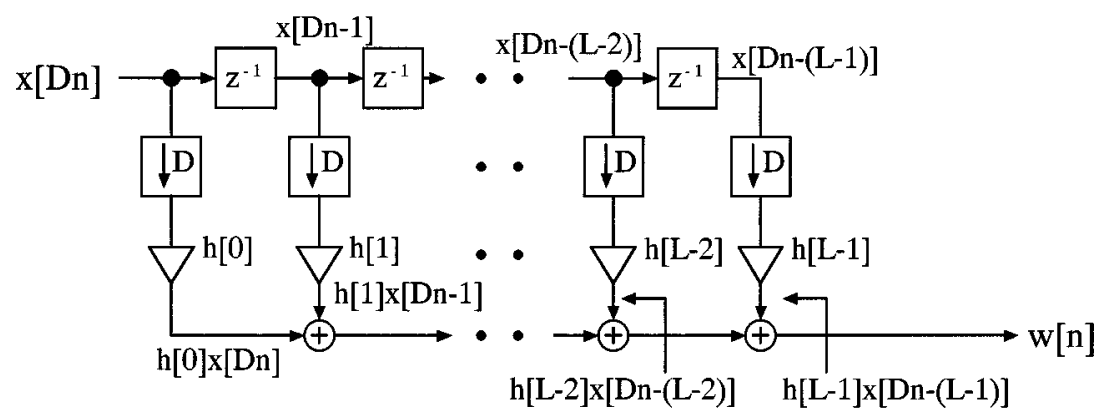

Fig. 9. Decimation by digital filter.

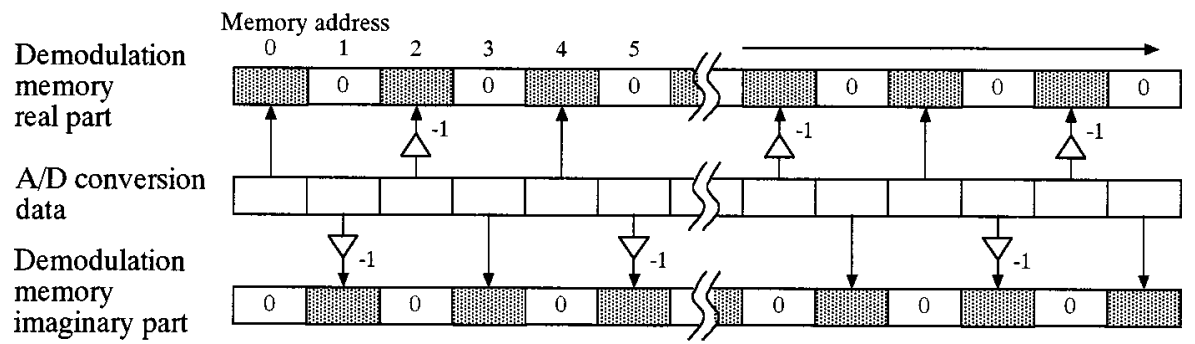

Fig. 10. Frequency spectrum shifting of A/D-converted data.

the frequency spectrum obtained by multiplying the data sequence $x[n]$ by the discrete complex exponential function $c[n]$ is

$$
X_{\text {shift }}(\Omega)=\sum_{n=-\infty}^{\infty}(x[n] \cdot c[n]) e^{-j \Omega n}=X\left(\Omega+\Omega_{C}\right) .
$$

Equation (11) indicates that the frequency spectrum $X(\Omega)$ has been shifted, or translated along the frequency axis.

Here, the discrete angular frequency $\Omega_{C}$ is

$$
\Omega_{C}=2 \pi \frac{f_{C}}{f_{S}}=2 \pi k+\frac{\pi}{2}
$$

where $k$ is an integer according to (5). Also from (8), we obtain

$$
\Omega_{C}=2 \pi \frac{f_{C}}{f_{S}}=2 \pi k+\frac{3 \pi}{2} .
$$

In the case of (12), the discrete complex exponential function $c[n]$ takes only the following four values regardless of the value of $n$ :

$$
c[n]=\left\{\begin{array}{lll}
e^{-j(\pi / 2) n}=+1, & & \mathrm{n}=4 \mathrm{~m} \\
e^{-j(\pi / 2) n}=-j, & & \mathrm{n}=4 \mathrm{~m}+1 \\
e^{-j(\pi / 2) n}=-1, & & \mathrm{n}=4 \mathrm{~m}+2 \\
e^{-j(\pi / 2) n}=+j, & & \mathrm{n}=4 \mathrm{~m}+3
\end{array}\right.
$$

where $m$ is an integer.

When (13) is used,

$$
c[n]=\left\{\begin{array}{lll}
e^{-j(3 \pi / 2) n}=+1, & & \mathrm{n}=4 \mathrm{~m} \\
e^{-j(3 \pi / 2) n}=+j, & & \mathrm{n}=4 \mathrm{~m}+1 \\
e^{-j(3 \pi / 2) n}=-1, & & \mathrm{n}=4 \mathrm{~m}+2 \\
e^{-j(3 \pi / 2) n}=-j, & & \mathrm{n}=4 \mathrm{~m}+3 .
\end{array}\right.
$$

It is recognized from the relationship between (14) and (15) that it is not necessary to actually perform multiplication of the

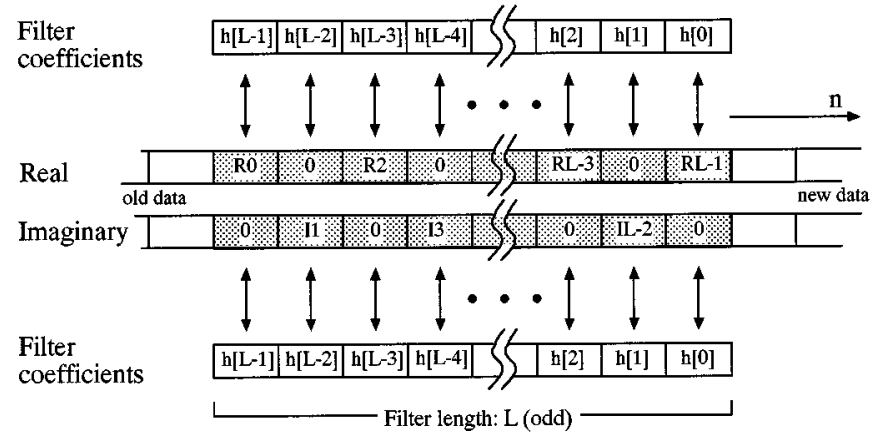

Fig. 11. Relationship between complex-valued data after frequency spectrum shifting and filter coefficients.

A/D-converted data by the complex exponential function for shifting the frequency spectrum.

In our alternative method, the shifting of the frequency spectrum is achieved by carrying out the following operations instead of performing multiplication. If the value of $c[n]$ is negative, the plus/minus sign of the A/D-converted data is inverted. If the value of $c[n]$ is real, the $\mathrm{A} / \mathrm{D}$-converted data is stored in a real data memory area for holding real parts and a zero is set in an imaginary data memory area for holding imaginary parts. On the contrary, if the value of $c[n]$ is imaginary, a zero is set in the real data memory area and the A/D-converted data is stored in the imaginary data memory area.

The frequency spectrum after undersampling is inverted in our example shown in Fig. 5. The inverted spectrum can be automatically returned to its original form by using (15) as the discrete exponential function.

\section{B. Decimation [13]}

Here, $x[n]$ is the original sampled sequence of discrete data. Let $x_{d}[n]$ represent a decimated data sequence obtained by extracting only every $D$ th sample of the sequence $x[n]$. There ex- 


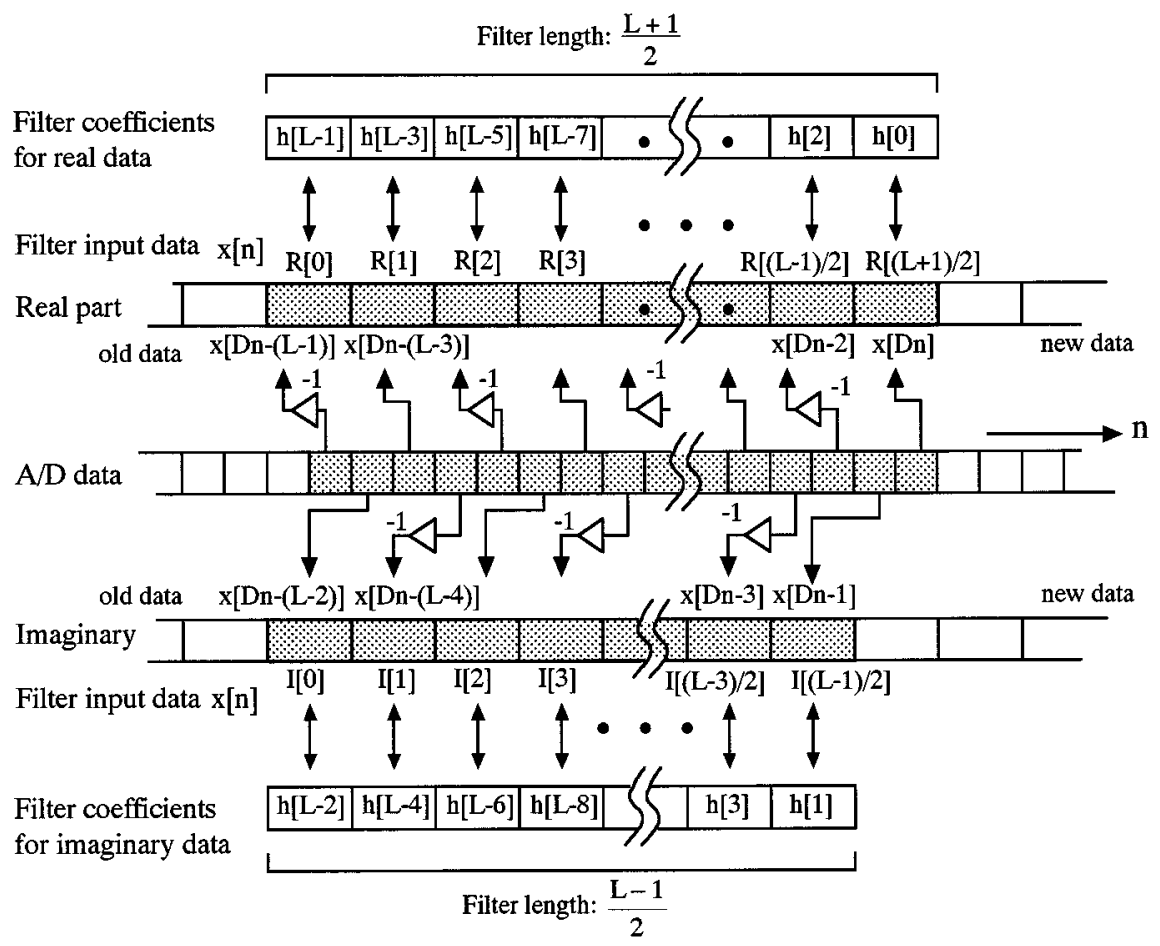

Fig. 12. Relationship between A/D-converted data, complex-valued data, and filter coefficients.

ists the following relationship between the sequences $x_{d}[n]$ and $x[n]$ :

$$
x_{d}[n]=x[n D] .
$$

Expressing the Fourier transforms of the sequences $x_{d}[n]$ and $x[n]$ as $X_{D}(\Omega)$ and $X(\Omega)$, respectively, we obtain

$$
X_{D}(\Omega)=\frac{1}{D} \sum_{k=0}^{D-1} X\left(\frac{\Omega}{D}-k \frac{2 \pi}{D}\right) .
$$

One can recognize from (4) to (9) that the spectrum has been expanded by a factor of $D$ along the frequency axis. If, however, the frequency components of the original discrete data sequence $x[n]$ are not bandlimited to satisfy $|\Omega|<\pi / D$, decimation will introduce aliasing. In this case, the original spectrum should usually be bandlimited by a lowpass filter prior to the decimation process to avoid aliasing.

Fig. 7 is a block diagram showing a sequence of operations for frequency spectrum zooming including a software mixer (frequency shifting), lowpass filtering, decimation, and FFT operation.

Fig. 8(a)-(c) shows changes in the spectrum pattern caused by the process of Fig. 7 .

Fig. 8(a) shows the spectrum obtained after the spectrum inversion has been eliminated by a $37.5-\mathrm{kHz}$ frequency shift. The data is lowpass-filtered prior to decimation to avoid aliasing. Spectra obtained after lowpass filtering and after subsequent decimation are depicted in Fig. 8(b) and (c), respectively. It can be seen from Fig. 8(a)-(c) that the real-valued sample data having a symmetrical spectrum are converted to complexvalued data having a single spectrum which falls in the sampling frequency band.
To achieve high-speed bandlimiting lowpass filtering and decimation operations with high efficiency when the decimation rate is $D$, these operations are to be performed on the real and imaginary parts of every $D$ th filter input only.

Fig. 9 is a block diagram illustrating a process of performing the filtering operation, skipping intermediate $D-1$ filter inputs, where $L$ represents the filter length.

\section{HIGH-SPEED PROCESSING}

When the frequency spectrum is shifted by multiplying it by the complex exponential function, every second real part, and every second imaginary part, of the complex-valued data produced by the multiplication are zero-valued as shown in Fig. 10.

Fig. 11 depicts the relationship between the complex-valued data after frequency spectrum shifting and filter coefficient, in which the filter length $L$ is an odd number.

Since multiplication of the filter coefficient by zero data does not affect the result of the filtering operation, it is possible to carry out the filtering operation without using any filter coefficient for the zero data. Therefore, the filtering operation can be performed by using the filter coefficients taken from a series of original filter coefficients alternately for the real and imaginary parts. This helps reduce execution time of the hardware and/or the software-based filtering operation by nearly half.

Based on the foregoing, the relationship between the A/D-converted data, the complex-valued data after frequency spectrum shifting, and the filter coefficient is shown in Fig. 12.

The same multipliers of the complex exponential function $c[n]$ recur for successive sets of four samples. Thus, if the decimation rate $D$ is set to an integer multiple of 4 , it is possible to multiply the filter coefficient by the complex exponential function beforehand. Then, it becomes possible to perform the fil- 


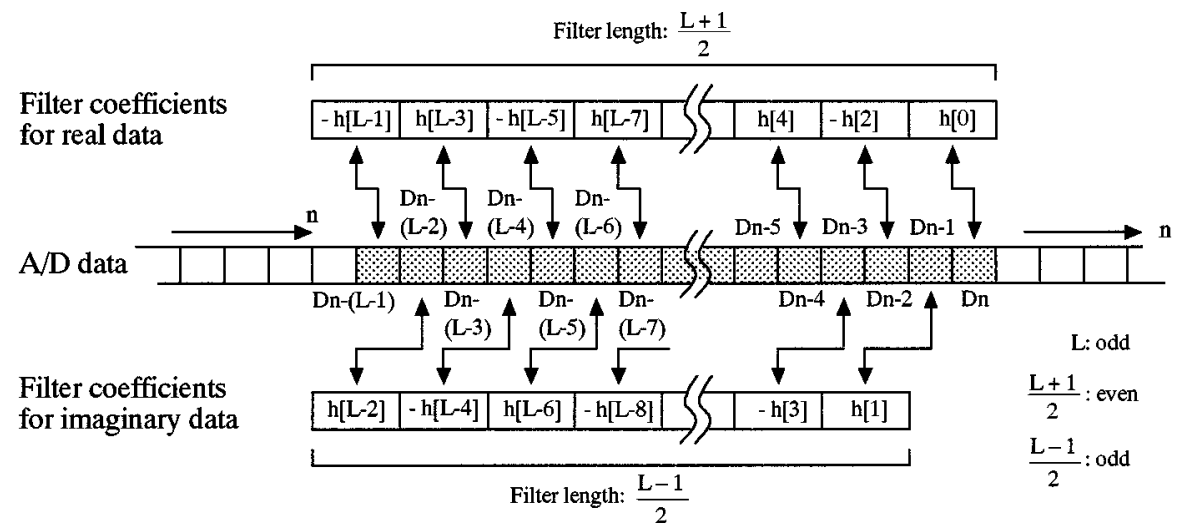

Fig. 13. A/D-converted data and filter coefficients for simultaneous execution of frequency spectrum shifting.
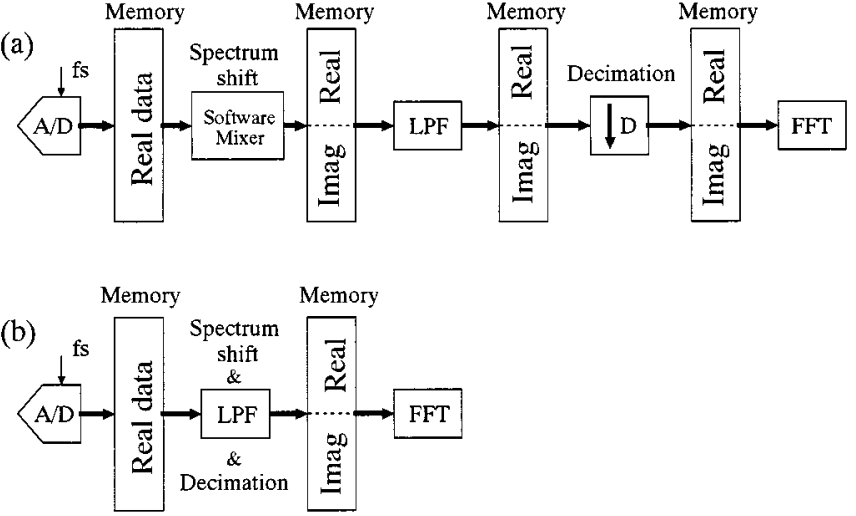

Fig. 14. Demodulation procedure and memory requirements. (a) Conventional sequence of the frequency spectrum shifting, filtering, and decimation operations. (b) Proposed sequence by the single filtering operation.

tering operation by directly multiplying the A/D-converted data by the filter coefficient as shown in Fig. 13.

Consequently, the frequency spectrum shifting, filtering, and decimation operations can be all together replaced by a single filtering process.

\section{Demodulation Procedure And Memory REQUIREMENTS}

Since frequency spectrum shifting, filtering, and decimation operations are performed in a single process, it is possible not only to execute mathematical operations, which have conventionally been performed separately, in a minimum of time, but also to eliminate excess memories for storing data derived from the individual operations. Fig. 14 illustrates operating procedures and memory requirements, in which Fig. 14(a) shows the case where the frequency spectrum shifting, filtering, and decimation operations are performed separately, and Fig. 14(b) shows the case where these operations are performed by the single filtering process.

The aforementioned one-time execution of the frequency spectrum shifting, filtering, and decimation operations permits us to take advantage of oversampling by sampling the data at relatively high frequencies where undersampling is possible. This also makes it possible to reduce quantization noise caused by the sampling operation.

\section{CONCLUSION}

We have examined the undersampling technique, wherein the echo signal containing the carrier frequency component from a transducer is A/D-converted and directly demodulated without using the mixer, and demonstrated the possibility of using this undersampling technique in frequency measurement by the Doppler sonar. To achieve this by hardware and DSP software, we have also examined the feasibility of a real-time system for high-accuracy CW Doppler frequency measurement with a simple configuration which performs the single filtering process combining the complex exponential function, filtering, and decimation operations.

Since the receiver demodulator proposed in the present paper can also be used in pulse-Doppler measurement, it is applicable to conventional pulse-Doppler algorithms as well without any change in the proposed circuit configuration.

\section{REFERENCES}

[1] H. Susaki, "Application of the continuous-wave Doppler measuring technique to flowmeters," in Proc. Seminar on Flow Measurements Using Ultrasound Doppler Methods (USDJ'97), Yokohama, Japan, 1997, pp. 70-84.

[2] Analog Devices Conversion Data Book, Analog Devices, Norwood, MA, 1997/1998.

[3] Burr-Brown Products Data Book, Texas Instruments, Dallas, TX, 1998/99.

[4] Analog Devices, System Applications Guide. Norwood, MA: Analog Devices, 1993, sec. XV.

[5] A. V. Oppenheim and R. W. Schafer, Digital Signal Processing. Englewood Cliffs, NJ: Prentice-Hall, 1975.

[6] A. V. Oppenheim, A. S. Willsky, and I. T. Young, Signals and Systems. Englewood Cliffs, NJ: Prentice-Hall, 1983.

[7] E. Del Re, "Bandpass signal filtering and reconstruction through minimum-sampling-rate digital processing," Alta Frequenza, vol. XLVII, no. 9, pp. 675-678, 1978 .

[8] D. W. Rice and K. H. Wu, "Quadrature sampling with high dynamic range," IEEE Trans. Aerosp. Electron. Syst., vol. AES-18, pp. 736-739, 1982.

[9] W. Rosenkranz, "Quadrature sampling of FM-bandpass signals," in Proc. Digital Signal Processing-87, 1987, pp. 377-381.

[10] C. B. Feldman and W. R. Bennett, "Band width and transmission performance," Bell Syst. Tech. J., vol. 28, pp. 490-595, 1949.

[11] P. F. Panter, Modulation, Noise and Spectral Analysis. New York: McGraw-Hill, 1965.

[12] H. Meyr, M. Moeneclaey, and S. A. Fechtel, Digital Communication Receivers. New York: Wiley-Interscience, 1998.

[13] A. V. Oppenheim, A. S. Willsky, and I. T. Young, Signals and Systems. Englewood Cliffs, NJ: Prentice-Hall, 1983. 


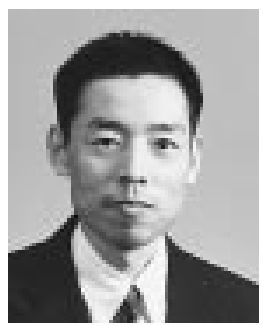

Hironori Susaki (M'89) was born in Tottori-city, Tottori, Japan, in 1952. He received the B.Sc., M.Sc., and Ph.D. degrees in applied physics from the University of Osaka, Osaka, Japan, in 1976, 1978 , and 1984, respectively.

From 1984 to 1986 , he was a part-time instructor in the medical school of the University of Tokushima, Tokushima, Japan. Since January 1985, he has been with Furuno Electric, Company, Ltd., Nishinomiya, Japan. His research interests are in the area of digital signal processing, image processing, digital communication system and micro-TAS 